\title{
APPROXIMATELY BIPROJECTIVE BANACH ALGEBRAS AND NILPOTENT IDEALS
}

\author{
HASAN POURMAHMOOD-AGHABABA
}

\author{
(Received 6 March 2012)
}

\begin{abstract}
By introducing a new notion of approximate biprojectivity we show that nilpotent ideals in approximately amenable or pseudo-amenable Banach algebras, and nilpotent ideals with the nilpotency degree larger than two in biflat Banach algebras cannot have the special property which we call "property $(\mathbb{B})^{\prime}$ (Definition 5.2 below) and hence, as a consequence, they cannot be boundedly approximately complemented in those Banach algebras.
\end{abstract}

2010 Mathematics subject classification: primary 46H20; secondary 46B28.

Keywords and phrases: Banach algebras, bounded approximately complemented subspaces, nilpotent ideals, approximate amenability, pseudo-amenability, approximate biprojectivity.

\section{Introduction}

In 1989, Curtis and Loy showed that an amenable Banach algebra cannot have a nonzero, finite-dimensional radical, and so nonzero nilpotent ideals in amenable Banach algebras must be infinite dimensional [2]. In 1994, Loy and Willis investigated the relation between existence of the approximation property and existence of nilpotent ideals in amenable and biprojective Banach algebras [13]. In particular, they proved the following two propositions.

Proposition 1.1. Let $\mathcal{A}$ be a biprojective Banach algebra with a central approximate identity, and let $\mathcal{N}$ be a nonzero nilpotent ideal in $\mathcal{A}$. Then $\mathcal{N}$ does not have the approximation property.

Proposition 1.2. Let $\mathcal{A}$ be an amenable Banach algebra and suppose that $\mathcal{N}$ is an ideal in $\mathcal{A}$ which is nilpotent, has the approximation property, and is such that multiplication on the left by any element of $\mathcal{N}$ is a compact operator on $\mathcal{A}$. Then $\mathcal{N}=\{0\}$.

At the end of the earlier paper, the authors conjectured that the biprojectivity condition in Proposition 1.1 or the compactness condition in Proposition 1.2 is not necessary. Related to these conjectures, in 1999, Zhang defined concepts of approximately complemented subspaces of normed spaces and approximately

This research was in part supported by a grant from IPM (No. 90470033).

(C) 2012 Australian Mathematical Publishing Association Inc. 0004-9727/2012 \$16.00 
biprojective Banach algebras. Using these concepts he proved that any approximately biprojective Banach algebra with left and right approximate identities does not have a nontrivial nilpotent ideal whose closure is approximately complemented [17].

In this paper, first we introduce a new notion of (bounded) approximate biprojectivity and determine its relation to other notions of approximate biprojectivity defined in [1, 17]. Then we characterise (bounded) approximate biprojectivity of some concrete Banach algebras, in particular, Banach algebras related to locally compact groups. Finally, we give another property of nilpotent ideals related to the conjectures raised by Loy and Willis in [13]. This property allows us to generalise results in $[13,17]$ to a large class of Banach algebras containing approximately amenable, pseudo-amenable and biflat Banach algebras. In particular, we show that in approximately biprojective Banach algebras, nilpotent ideals cannot have a spacial property, called the 'property (B)' (Definition 5.2). As a consequence, we show that they cannot be boundedly approximately complemented in those Banach algebras. We also show that in biflat Banach algebras, nilpotent ideals with the nilpotency degree larger than two cannot be boundedly approximately complemented.

\section{Preliminaries}

For two Banach spaces $\mathcal{X}$ and $\mathcal{Y}$, we denote by $\mathcal{X} \hat{\otimes} \mathcal{Y}$ the projective tensor product of them, and by $\mathcal{B}(\mathcal{X}, \mathcal{Y})$ the space of all bounded linear operators from $\mathcal{X}$ to $\mathcal{Y}$. Suppose that $\mathcal{X}_{1}, \mathcal{X}_{2}, \mathcal{Y}_{1}$ and $\mathcal{Y}_{2}$ are Banach spaces and $T_{i} \in \mathcal{B}\left(\mathcal{X}_{i}, \mathcal{Y}_{i}\right), i=1,2$. The tensor product of $T_{1}$ and $T_{2}$ is the linear map defined by $T_{1} \otimes T_{2}: \mathcal{X}_{1} \hat{\otimes} \mathcal{X}_{2} \rightarrow \mathcal{Y}_{1} \hat{\otimes} \mathcal{Y}_{2}$ by

$$
\left(T_{1} \otimes T_{2}\right)\left(x_{1} \otimes x_{2}\right)=T_{1}\left(x_{1}\right) \otimes T_{2}\left(x_{2}\right) \quad\left(x_{1} \in \mathcal{X}_{1}, x_{2} \in \mathcal{X}_{2}\right) .
$$

For two Banach spaces $\mathcal{X}$ and $\mathcal{Y}$ we say that the net $\left(T_{i}\right) \subset \mathcal{B}\left(\mathcal{X}, \mathcal{Y}^{*}\right)$ converges to $T$ in the weak* operator topology of $\mathcal{B}\left(\mathcal{X}, \mathcal{Y}^{*}\right)$ if $T_{i}(x) \rightarrow T(x)$ in the $w^{*}$-topology of $\mathcal{Y}^{*}$, for all $x \in \mathcal{X}$, where $\mathcal{Y}^{*}$ is the dual space of $\mathcal{Y}$.

A Banach space $\mathcal{X}$ is said to have the (bounded) approximation property if there exists a (bounded) net $\left(T_{\alpha}\right)$ of finite rank operators on $\mathcal{X}$ such that $T_{\alpha} \rightarrow I_{\mathcal{X}}$ uniformly on compact subsets of $\mathcal{X}$, where $I_{\mathcal{X}}$ is the identity map on $\mathcal{X}$.

A very close and related notion to the (bounded) approximation property is (bounded) approximately complementation introduced and studied by Zhang in $[17,18]$.

A subspace $\mathcal{Y}$ of a normed space $\mathcal{X}$ is called (bounded) approximately complemented in $\mathcal{X}$ if there is a (bounded) net $\left(P_{\alpha}\right)$ of continuous operators from $\mathcal{X}$ into $\mathcal{Y}$ such that $P_{\alpha}(x)$ converges to $x$ uniformly on compact subsets of $\mathcal{Y}$.

Let $\mathcal{A}$ be a Banach algebra. The product map on $\mathcal{A}$ extends to a map $\Delta: \mathcal{A} \hat{\otimes} \mathcal{A} \rightarrow$ $\mathcal{A}$, determined by $\Delta(a \otimes b)=a b$ for all $a, b \in \mathcal{A}$. The projective tensor product $\mathcal{A} \hat{\otimes} \mathcal{A}$ becomes a Banach $\mathcal{A}$-bimodule with the following module actions:

$$
a \cdot(b \otimes c)=a b \otimes c, \quad(b \otimes c) \cdot a=b \otimes c a \quad(a, b, c \in \mathcal{A}) .
$$

With the above actions $\Delta$ becomes an $\mathcal{A}$-bimodule homomorphism. 
A Banach algebra $\mathcal{A}$ is called biprojective if $\Delta$ has a bounded right inverse which is an $\mathcal{A}$-bimodule homomorphism, and is called biflat if $\Delta^{*}$ has a bounded left inverse which is an $\mathcal{A}$-bimodule homomorphism. An ideal $\mathcal{I}$ of $\mathcal{A}$ is called nilpotent if there is a positive integer $n$ such that $\mathcal{I}^{n}=0$. The smallest such $n$, denoted by $\operatorname{Nil}(\mathcal{I})$, will be termed the nilpotency degree of $\mathcal{I}$.

The unitisation of $\mathcal{A}$ is denoted by $\mathcal{A}^{\#}$, where $\mathcal{A}^{\#}=\mathcal{A} \oplus \mathbb{C}$ with the following product:

$$
(a, \lambda) \cdot(b, \mu)=(a b+\mu a+\lambda b, \lambda \mu) \quad(a, b \in \mathcal{A}, \lambda, \mu \in \mathbb{C}) .
$$

It is obvious that $\mathcal{A}^{\#}$ is a Banach algebra as well.

Let $\mathcal{A}$ be a Banach algebra and $\mathcal{X}$ a Banach $\mathcal{A}$-bimodule. Then $\mathcal{X}^{*}$ is a Banach $\mathcal{A}$-bimodule in the canonical way. A derivation from $\mathcal{A}$ into $\mathcal{X}$ is a bounded linear map $D: \mathcal{A} \rightarrow \mathcal{X}$ such that $D(a b)=a \cdot D(b)+D(a) \cdot b$ for all $a, b \in \mathcal{A}$. For each $x \in \mathcal{X}$, the derivation $D(a)=a \cdot x-x \cdot a$ is termed an inner derivation and denoted by $\operatorname{ad}_{x}$. A Banach algebra $\mathcal{A}$ is called amenable if every derivation $D: \mathcal{A} \rightarrow \mathcal{X}^{*}$ is inner for each Banach $\mathcal{A}$-bimodule $\mathcal{X}$, and is called contractible if each derivation $D: \mathcal{A} \rightarrow \mathcal{X}$ is inner for each Banach $\mathcal{A}$-bimodule $\mathcal{X}$. A derivation $D: \mathcal{A} \rightarrow \mathcal{X}$ is called approximately inner if there exists a net $\left(x_{\alpha}\right) \subseteq \mathcal{X}$ such that $D(a)=\lim _{\alpha} \operatorname{ad}_{x_{\alpha}}(a)$ for all $a \in \mathcal{A}$. A Banach algebra $\mathcal{A}$ is approximately amenable if every derivation $D: \mathcal{A} \rightarrow \mathcal{X}^{*}$ is approximately inner.

A Banach algebra $\mathcal{A}$ is pseudo-amenable if there is a net $\left(\mathbf{m}_{\alpha}\right) \subseteq \mathcal{A} \hat{\otimes} \mathcal{A}$, called an approximate diagonal, such that $a \cdot \mathbf{m}_{\alpha}-\mathbf{m}_{\alpha} \cdot a \rightarrow 0$ and $\left(\Delta \mathbf{m}_{\alpha}\right) a \rightarrow a$ for each $a \in \mathcal{A}$, and is pseudo-contractible if it has a central approximate diagonal, that is, an approximate diagonal $\left(\mathbf{m}_{\alpha}\right)$ satisfying $a \cdot \mathbf{m}_{\alpha}=\mathbf{m}_{\alpha} \cdot a$ for all $a \in \mathcal{A}$. It is known that a Banach algebra $\mathcal{A}$ is approximately amenable if and only if $\mathcal{A}^{\#}$ is pseudo-amenable [10, Theorem 3.1].

\section{Approximately biprojective Banach algebras}

The present paper is based on the following definition.

Definition 3.1. A Banach algebra $\mathcal{A}$ is called approximately biprojective if there exists a net $\left(\rho_{\alpha}\right) \subseteq \mathcal{B}(\mathcal{A}, \mathcal{A} \hat{\otimes} \mathcal{A})$ such that:

(i) $\Delta \circ \rho_{\alpha}(a) \rightarrow a$ for all $a \in \mathcal{A}$;

(ii) $a \cdot \rho_{\alpha}(b)-\rho_{\alpha}(a b) \rightarrow 0$ for all $a, b \in \mathcal{A}$;

(iii) $\rho_{\alpha}(a) \cdot b-\rho_{\alpha}(a b) \rightarrow 0$ for all $a, b \in \mathcal{A}$.

Moreover, if the net $\left(\rho_{\alpha}\right)$ can be chosen bounded, we say that $\mathcal{A}$ is boundedly approximately biprojective.

Note that a different notion of approximate biprojectivity is considered in $[1,17]$. Zhang assumes that each $\rho_{\alpha}$ in Definition 3.1 is an $\mathcal{A}$-bimodule homomorphism and Aristov assumes that the convergence in conditions (i), (ii) and (iii) is the uniform convergence on compact subsets of $\mathcal{A}$ (see [1, Proposition 5.5]). Of course, bounded approximate biprojectivity is the same as Aristov's one; see [1, Theorem 7.2]. By [10, 
Proposition 3.8], approximate biprojectivity of a unital Banach algebra, in the sense of Zhang, is equivalent to the pseudo-contractibility of it. But by [10, Theorem 2.4], pseudo-contractibility of a unital Banach algebra is equivalent to the contractibility. Therefore, unital approximately biprojective Banach algebras, with the definition of Zhang, are not interesting.

We now proceed to give examples of approximately biprojective Banach algebras. We show that there are large classes of unital approximately biprojective, noncontractible Banach algebras that distinguish our definition from Zhang's. First note that, trivially, every approximately biprojective Banach algebra in the sense of Zhang or Aristov (in particular, every biprojective Banach algebra) is approximately biprojective (in the sense of Definition 3.1). Moreover:

(i) every biflat Banach algebra with the approximation property is approximately biprojective [1, Theorem 3.6(B)]; of course, we will show that, based on Definition 3.1, every biflat Banach algebra (with or without the approximation property) is approximately biprojective (see Theorem 3.3);

(ii) every biflat Banach algebra with a bounded one-sided approximate identity is boundedly approximately biprojective [1, Proposition 7.5]; in particular, every amenable Banach algebra is boundedly approximately biprojective;

(iii) every boundedly approximately biprojective Banach algebra is biflat [1, Theorem 3.6(A)];

(iv) every biflat Banach algebra with the bounded approximation property is boundedly approximately biprojective [1, Theorem 7.3(C)].

For two Banach spaces $\mathcal{X}$ and $\mathcal{Y}$ we denote by $\mathcal{F}(\mathcal{X}, \mathcal{Y})$ the space of finite rank operators from $\mathcal{X}$ to $\mathcal{Y}$. We also denote by $F(\mathcal{X})$ the set of all finite subsets of $\mathcal{X}$. The cardinality of a set $K$ will be denoted by $\# K$.

Lemma 3.2. Let $\mathcal{X}$ and $\mathcal{Y}$ be Banach spaces. Then $\mathcal{F}\left(\mathcal{X}, \mathcal{Y}^{*}\right)$ is dense in $\mathcal{B}\left(\mathcal{X}, \mathcal{Y}^{*}\right)$ with respect to the weak* operator topology.

Proof. Let $T \in \mathcal{B}\left(\mathcal{X}, \mathcal{Y}^{*}\right)$ and take a finite subset $F=\left\{x_{1}, \ldots, x_{n}\right\}$ of $\mathcal{X}$. Assume that $x_{1}, \ldots, x_{m}$ are linearly independent $(m \leq n)$. By the Hahn Banach theorem, for each $j \in\{1, \ldots, m\}$, there is $f_{j} \in \mathcal{X}^{*}$ such that $f_{j}\left(x_{j}\right)=1$ and $f_{j}\left(x_{i}\right)=0$ for all $i \in$ $\{1, \ldots, m\} \backslash\{j\}$. For each $j \in\{1, \ldots, m\}$ define $T_{j} \in \mathcal{B}\left(\mathcal{X}, \boldsymbol{Y}^{*}\right)$ by $T_{j}(x)=f_{j}(x) T\left(x_{j}\right)$. Then

$$
T_{j}\left(x_{j}\right)=T\left(x_{j}\right), \quad T_{j}\left(x_{i}\right)=0 \quad(i, j \in\{1, \ldots, m\}, i \neq j) .
$$

Now define $T_{F}=T_{1}+\cdots+T_{m}$. It can be easily seen that

$$
T_{F}\left(x_{i}\right)=T\left(x_{i}\right) \quad(i \in\{1, \ldots, n\}) .
$$

So $T_{F}=T$ on the span of $F$ and $\operatorname{rank}\left(T_{F}\right) \leq \# F$. Now it is obvious that the net $\left(T_{F}\right)_{F \in F(X)}$ converges to $T$ in the weak* operator topology, as desired. 
The following theorem is proved with a similar argument akin to [8, Theorem 2.1]. Theorem 3.3. Let $\mathcal{A}$ be a biflat Banach algebra. Then $\mathcal{A}$ is approximately biprojective. Proof. By [12, Exercise VII.2.8], there is an $\mathcal{A}$-bimodule homomorphism $\tau \in$ $\mathcal{B}\left(\mathcal{A},(\mathcal{A} \hat{\otimes} \mathcal{A})^{* *}\right)$ such that $\Delta^{* *} \circ \tau$ is the canonical embedding of $\mathcal{A}$ into $\mathcal{A}^{* *}$. By Lemma 3.2 and the principal of local reflexivity [15, Theorem C.3.3], there is a net $\left(\tau_{\lambda}\right) \subset \mathcal{F}(\mathcal{A}, \mathcal{A} \hat{\otimes} \mathcal{A})$ such that $\tau_{\lambda} \rightarrow \tau$ in the weak* operator topology of $\mathcal{B}\left(\mathcal{A},(\mathcal{A} \hat{\otimes} \mathcal{A})^{* *}\right)$; equivalently, $\tau_{\lambda}(a) \rightarrow \tau(a)$ in the weak* topology of $(\mathcal{A} \hat{\otimes} \mathcal{A})^{* *}$ for all $a \in \mathcal{A}$. So, for each $a, b \in \mathcal{A}$,

$$
\tau_{\lambda}(a b)-a \cdot \tau_{\lambda}(b) \rightarrow 0 \quad \text { and } \quad \tau_{\lambda}(a b)-\tau_{\lambda}(a) \cdot b \rightarrow 0 \text { weakly in } \mathcal{A} \hat{\otimes} \mathcal{A},
$$

and

$$
\Delta \circ \tau_{\lambda}(a) \rightarrow a \text { weakly in } \mathcal{A} \text {. }
$$

Now take $\epsilon>0$ and two finite subsets $F=\left\{a_{1}, \ldots, a_{n}\right\}$ and $G=\left\{b_{1}, \ldots, b_{n}\right\}$ of $\mathcal{A}$, and set

$$
\begin{aligned}
V & =\left\{\left(\rho\left(a_{i} b_{i}\right)-a_{i} \cdot \rho\left(b_{i}\right), \rho\left(a_{i} b_{i}\right)-\rho\left(a_{i}\right) \cdot b_{i}, \Delta \circ \rho\left(a_{i}\right)-a_{i}\right) \mid \rho \in \mathcal{B}(\mathcal{A}, \mathcal{A} \hat{\otimes} \mathcal{A})\right\}_{i=1, \ldots, n} \\
& \subseteq(\mathcal{A} \hat{\otimes} \mathcal{A})^{2 n} \oplus \mathcal{A}^{n}
\end{aligned}
$$

Then $V$ is a convex set and thus the weak and norm closures of $V$ coincide by Mazur's theorem. Thus, by (3.1) and (3.2), (0, . , 0) belongs to the norm closure of $V$. Hence, there is a $\rho_{(F, G, \epsilon)} \in \mathcal{B}(\mathcal{A}, \mathcal{A} \hat{\otimes} \mathcal{A})$ such that

$$
\left\|\rho_{(F, G, \epsilon)}\left(a_{i} b_{i}\right)-a_{i} \cdot \rho_{(F, G, \epsilon)}\left(b_{i}\right)\right\|<\epsilon, \quad\left\|\rho_{(F, G, \epsilon)}\left(a_{i} b_{i}\right)-\rho_{(F, G, \epsilon)}\left(a_{i}\right) \cdot b_{i}\right\|<\epsilon,
$$

and

$$
\left\|\Delta \circ \rho_{(F, G, \epsilon)}\left(a_{i}\right)-a_{i}\right\|<\epsilon,
$$

for each $i \in\{1, \ldots, n\}$. Now consider the directed set

$$
\Gamma=\{(F, G, \epsilon): F, G \in F(\mathcal{A}), \# F=\# G, \epsilon>0\},
$$

with the order

$$
(F, G, \epsilon) \leq\left(F^{\prime}, G^{\prime}, \epsilon^{\prime}\right) \Longleftrightarrow F \subseteq F^{\prime}, G \subseteq G^{\prime} \text { and } \epsilon \geq \epsilon^{\prime}
$$

Then the net $\left(\rho_{(F, G, \epsilon)}\right)_{(F, G, \epsilon) \in \Gamma}$ is as required, and $\mathcal{A}$ is approximately biprojective.

Now we are going to determine the relationship between some notions of amenability and approximate biprojectivity. The following proposition is an easy observation which is also proved in [16, Theorem 2.4].

Proposition 3.4. Let $\mathcal{A}$ be an approximately amenable or a pseudo-amenable Banach algebra. Then $\mathcal{A}$ is approximately biprojective. 
Proof. Let $\mathcal{A}$ be approximately amenable. Then by [8, Theorem 2.1] and [7, Proposition 2.6] there are nets $\left(\mathbf{m}_{\alpha}\right) \subseteq \mathcal{A} \hat{\otimes} \mathcal{A},\left(F_{\alpha}\right),\left(G_{\alpha}\right) \subseteq \mathcal{A}$ such that:

(i) $a \cdot \mathbf{m}_{\alpha}-\mathbf{m}_{\alpha} \cdot a+F_{\alpha} \otimes a-a \otimes G_{\alpha} \rightarrow 0$;

(ii) $a F_{\alpha} \rightarrow a, G_{\alpha} a \rightarrow a$; and

(iii) $\Delta\left(\mathbf{m}_{\alpha}\right) a-F_{\alpha} a-G_{\alpha} a \rightarrow 0$.

Define $\rho_{\alpha}: \mathcal{A} \rightarrow \mathcal{A} \hat{\otimes} \mathcal{A}$ by $\rho_{\alpha}(a)=\mathbf{m}_{\alpha} \cdot a-F_{\alpha} \otimes a$ for all $a \in \mathcal{A}$. Let $a, b \in \mathcal{A}$. Then obviously $\rho_{\alpha}(a b)=\rho_{\alpha}(a) \cdot b$. Now, using (i),

$$
\begin{aligned}
\lim _{\alpha} \rho_{\alpha}(a b)-a \cdot \rho_{\alpha}(b) & =\lim _{\alpha} \mathbf{m}_{\alpha} \cdot a b-F_{\alpha} \otimes a b-a \cdot \mathbf{m}_{\alpha} \cdot b+a F_{\alpha} \otimes b \\
& =\lim _{\alpha} \mathbf{m}_{\alpha} \cdot a b-F_{\alpha} \otimes a b-a b \cdot \mathbf{m}_{\alpha}+a b \otimes G_{\alpha} \\
& =0,
\end{aligned}
$$

and, using (ii), (iii),

$$
\lim _{\alpha} \Delta \circ \rho_{\alpha}(a)=\lim _{\alpha}\left(\Delta \mathbf{m}_{\alpha}\right) a-F_{\alpha} a=\lim _{\alpha} G_{\alpha} a=a .
$$

Hence, $\mathcal{A}$ is approximately biprojective.

If $\mathcal{A}$ is pseudo-amenable with approximate diagonal $\left(\mathbf{m}_{\alpha}\right)$, then the net $\left(\rho_{\alpha}\right)$ with $\rho_{\alpha}(a)=a \cdot \mathbf{m}_{\alpha}$ satisfies Definition 3.1.

We remark that the converse of Proposition 3.4 does not hold in general. For example, $l^{1}$ (the Banach algebra of all sequences $\left(a_{n}\right) \subset \mathbb{C}$ such that $\left\|\left(a_{n}\right)\right\|=\sum_{n=1}^{\infty}\left|a_{n}\right|<$ $\infty$, with pointwise product) is a biprojective Banach algebra but not approximately amenable [5].

Similarly to Proposition 3.8 of [10], the following proposition is a partial converse for the above proposition in the case of pseudo-amenability.

Proposition 3.5. Let $\mathcal{A}$ be an approximately biprojective Banach algebra with a central approximate identity. Then $\mathcal{A}$ is pseudo-amenable.

Proof. Let $\left(\rho_{\alpha}\right)$ be a net satisfying Definition 3.1 and let $\left(e_{\beta}\right)$ be a central approximate identity for $\mathcal{A}$. Take $\epsilon>0$ and let $F$ be a finite subset of $\mathcal{A}$. Set $M=\sum_{a \in F}\|a\|$. There is a $\beta=\beta(F, \epsilon)$ such that $\left\|e_{\beta} a-a\right\|<\epsilon / 2$ for all $a \in F$. Now let $\alpha=\alpha(F, \epsilon)$ be such that

$$
\left\|\Delta \circ \rho_{\alpha}\left(e_{\beta}\right)-e_{\beta}\right\|<\frac{\epsilon}{2 M+1},
$$

and, for $a \in F$,

$$
\left\|a \cdot \rho_{\alpha}\left(e_{\beta}\right)-\rho_{\alpha}\left(a e_{\beta}\right)\right\|<\frac{\epsilon}{2} \quad \text { and } \quad\left\|\rho_{\alpha}\left(e_{\beta}\right) \cdot a-\rho_{\alpha}\left(e_{\beta} a\right)\right\|<\frac{\epsilon}{2} .
$$

Then

$$
\left\|\Delta \circ \rho_{\alpha}\left(e_{\beta}\right) a-a\right\| \leq\left\|\Delta \circ \rho_{\alpha}\left(e_{\beta}\right) a-e_{\beta} a\right\|+\left\|e_{\beta} a-a\right\|<\frac{M \epsilon}{2 M+1}+\frac{\epsilon}{2}<\epsilon .
$$

Now set $\mathbf{m}_{(F, \epsilon)}=\rho_{\alpha(F, \epsilon)}\left(e_{\beta(F, \epsilon)}\right)$ with the order

$$
(F, \epsilon) \leq\left(F^{\prime}, \epsilon^{\prime}\right) \Longleftrightarrow F \subseteq F^{\prime} \text { and } \epsilon \geq \epsilon^{\prime} \text {. }
$$


Obviously, $\lim _{(F, \epsilon)} \Delta\left(\mathbf{m}_{(F, \epsilon)}\right) a=a$ for $a \in \mathcal{A}$. For each $a \in \mathcal{A}$ we also have

$$
\begin{aligned}
&\left\|a \cdot \mathbf{m}_{(F, \epsilon)}-\mathbf{m}_{(F, \epsilon)} \cdot a\right\|=\left\|a \cdot \rho_{\alpha(F, \epsilon)}\left(e_{\beta(F, \epsilon)}\right)-\rho_{\alpha(F, \epsilon)}\left(e_{\beta(F, \epsilon)}\right) \cdot a\right\| \\
& \leq\left\|a \cdot \rho_{\alpha(F, \epsilon)}\left(e_{\beta(F, \epsilon)}\right)-\rho_{\alpha(F, \epsilon)}\left(a e_{\beta(F, \epsilon)}\right)\right\| \\
& \quad \quad+\left\|\rho_{\alpha(F, \epsilon)}\left(e_{\beta(F, \epsilon)} a\right)-\rho_{\alpha(F, \epsilon)}\left(e_{\beta(F, \epsilon)}\right) \cdot a\right\| \\
&<\epsilon,
\end{aligned}
$$

if $a \in F$. Hence, for $a \in \mathcal{A}, \lim _{(F, \epsilon)}\left(a \cdot \mathbf{m}_{(F, \epsilon)}-\mathbf{m}_{(F, \epsilon)} \cdot a\right)=0$, and so $\left(\mathbf{m}_{(F, \epsilon)}\right)$ makes an approximate diagonal for $\mathcal{A}$.

Corollary 3.6. Let $\mathcal{A}$ be a Banach algebra with a central approximate identity. Then $\mathcal{A}$ is approximately biprojective if and only if it is pseudo-amenable.

Proof. Use Propositions 3.4 and 3.5.

Corollary 3.7. Let $\mathcal{A}$ be a Banach algebra with a central bounded approximate identity. The following are equivalent:

(i) $\mathcal{A}$ is approximately biprojective;

(ii) $\mathcal{A}$ is pseudo-amenable;

(iii) $\mathcal{A}$ is approximately amenable.

Proof. By Corollary 3.6, statements (i) and (ii) are equivalent. The equivalence of (ii) and (iii) is proved in [10, Proposition 3.2].

Therefore, for unital Banach algebras, the three phrases 'approximate biprojectivity', 'pseudo-amenability' and 'approximate amenability' are the same. This shows that our definition of approximate biprojectivity is different from Zhang's and covers many examples of Banach algebras.

As a consequence of Corollary 3.7 we obtain the following.

Corollary 3.8. A Banach algebra $\mathcal{A}$ is approximately amenable if and only if $\mathcal{A}^{\#}$ is approximately biprojective.

Another partial converse to the Proposition 3.4 in the case of pseudo-amenability is the following proposition. The proof is similar to the proof of Proposition 3.5.

Proposition 3.9. Let $\mathcal{A}$ be a boundedly approximately biprojective Banach algebra with an approximate identity. Then $\mathcal{A}$ is pseudo-amenable.

Proof. Let $N=\sup _{\alpha}\left\|\rho_{\alpha}\right\|$, where $\left(\rho_{\alpha}\right)$ satisfies Definition 3.1. Take $\epsilon>0$ and let $F$ be a finite subset of $\mathcal{A}$, and $M=\sum_{a \in F}\|a\|$. There is a $\beta=\beta(F, \epsilon)$ such that

$$
\left\|e_{\beta(F, \epsilon)} a-a\right\|<\frac{\epsilon}{2 N+2} \quad \text { and } \quad\left\|a e_{\beta(F, \epsilon)}-a\right\|<\frac{\epsilon}{2 N+2},
$$

for all $a \in F$. Now let $\alpha=\alpha(F, \epsilon)$ be such that

$$
\left\|\Delta \circ \rho_{\alpha}\left(e_{\beta}\right)-e_{\beta}\right\|<\frac{\epsilon}{2 M+1},
$$


and, for $a \in F$,

$$
\left\|a \cdot \rho_{\alpha}\left(e_{\beta}\right)-\rho_{\alpha}\left(a e_{\beta}\right)\right\|<\frac{\epsilon}{2} \quad \text { and } \quad\left\|\rho_{\alpha}\left(e_{\beta}\right) \cdot a-\rho_{\alpha}\left(e_{\beta} a\right)\right\|<\frac{\epsilon}{2} .
$$

Then

$$
\left\|\Delta \circ \rho_{\alpha}\left(e_{\beta}\right) a-a\right\| \leq\left\|\Delta \circ \rho_{\alpha}\left(e_{\beta}\right) a-e_{\beta} a\right\|+\left\|e_{\beta} a-a\right\|<\frac{M \epsilon}{2 M+1}+\frac{\epsilon}{2 N+2}<\epsilon .
$$

As in Proposition 3.5, set $\mathbf{m}_{(F, \epsilon)}=\rho_{\alpha(F, \epsilon)}\left(e_{\beta(F, \epsilon)}\right)$ with the same order. We have only to check that $\lim _{(F, \epsilon)}\left(a \cdot \mathbf{m}_{(F, \epsilon)}-\mathbf{m}_{(F, \epsilon)} \cdot a\right)=0$. For each $a \in \mathcal{A}$,

$$
\begin{aligned}
&\left\|a \cdot \mathbf{m}_{(F, \epsilon)}-\mathbf{m}_{(F, \epsilon)} \cdot a\right\|=\left\|a \cdot \rho_{\alpha(F, \epsilon)}\left(e_{\beta(F, \epsilon)}\right)-\rho_{\alpha(F, \epsilon)}\left(e_{\beta(F, \epsilon)}\right) \cdot a\right\| \\
& \leq\left\|a \cdot \rho_{\alpha(F, \epsilon)}\left(e_{\beta(F, \epsilon)}\right)-\rho_{\alpha(F, \epsilon)}\left(a e_{\beta(F, \epsilon)}\right)\right\| \\
& \quad+\left\|\rho_{\alpha(F, \epsilon)}\left(a e_{\beta(F, \epsilon)}\right)-\rho_{\alpha(F, \epsilon)}\left(e_{\beta(F, \epsilon)} a\right)\right\| \\
& \quad+\left\|\rho_{\alpha(F, \epsilon)}\left(e_{\beta(F, \epsilon)} a\right)-\rho_{\alpha(F, \epsilon)}\left(e_{\beta(F, \epsilon)}\right) \cdot a\right\| \\
&<+N\left\|a e_{\beta(F, \epsilon)}-e_{\beta(F, \epsilon)} a\right\| \\
&<2 \epsilon .
\end{aligned}
$$

Therefore, $\lim _{(F, \epsilon)}\left(a \cdot \mathbf{m}_{(F, \epsilon)}-\mathbf{m}_{(F, \epsilon)} \cdot a\right)=0$.

The following corollary is also proved in [1, Theorem 7.1(B)].

Corollary 3.10. A Banach algebra $\mathcal{A}$ is amenable if and only if it is boundedly approximately biprojective and has a bounded approximate identity.

Proof. If $\left(e_{\beta}\right)$ is bounded in the proof of Proposition 3.9, $\left(\mathbf{m}_{(F, \epsilon)}\right)$ becomes a bounded approximate diagonal for $\mathcal{A}$, and so $\mathcal{A}$ is amenable by [15, Theorem 2.2.4]. For the converse, let $\left(\mathbf{m}_{\alpha}\right)$ be a bounded approximate diagonal for $\mathcal{A}$. As in the proof of Proposition 3.4, the bounded net $\left(\rho_{\alpha}\right)$ defined by $\rho_{\alpha}(a)=a \cdot \mathbf{m}_{\alpha}$ is bounded and satisfies Definition 3.1.

Now we give some properties of approximately biprojective Banach algebras. First note that every approximately biprojective Banach algebra must be essential (that is, $\overline{\mathcal{A}^{2}}=\mathcal{A}$, where $\mathcal{A}^{2}$ denotes the linear span of $\left.\{a b \mid a, b \in \mathcal{A}\}\right)$, since for $a \in \mathcal{A}$, $\Delta \circ \rho_{\alpha}(a) \in \overline{\mathcal{A}^{2}}$ and so $a$ belongs to $\overline{\mathcal{A}^{2}}$.

Proposition 3.11. Let $\mathcal{A}$ be an approximately biprojective Banach algebra. Then $\mathcal{A}$ is $w^{*}$-approximately weakly amenable (that is, for every derivation $D: \mathcal{A} \rightarrow \mathcal{A}^{*}$, there is a net $\left(f_{i}\right) \subset \mathcal{A}^{*}$ such that $D(a)=w^{*}-\lim _{i} \operatorname{ad}_{f_{i}}(a)$, for all $\left.a \in \mathcal{A}\right)$.

Proof. Let $\left(\rho_{\alpha}\right)$ be as in Definition 3.1. Clearly, for $a \in \mathcal{A}$ and $f \in \mathcal{A}^{*}$,

$$
\left(\rho_{\alpha}^{*} \circ \Delta^{*}\right)(f) \rightarrow f
$$

in the $w^{*}$-topology of $\mathcal{A}^{*}$. Moreover,

$$
a \cdot \rho_{\alpha}^{*}(f)-\rho_{\alpha}^{*}(a \cdot f) \rightarrow 0 \quad \text { and } \quad \rho_{\alpha}^{*}(f) \cdot a-\rho_{\alpha}^{*}(f \cdot a) \rightarrow 0,
$$


in the $w^{*}$-topology of $(\mathcal{A} \hat{\otimes} \mathcal{A})^{*}$. Now let $D: \mathcal{A} \rightarrow \mathcal{A}^{*}$ be a derivation. Define $f \in(\mathcal{A} \hat{\otimes} \mathcal{A})^{*}$ by $f(a \otimes b)=\langle b, D(a)\rangle$. Then, for $a, b, c \in \mathcal{A}$,

$$
\begin{aligned}
\langle b \otimes c, a \cdot f-f \cdot a\rangle & =\langle b \otimes c a-a b \otimes c, f\rangle=\langle c a, D(b)\rangle-\langle c, D(a b)\rangle \\
& =\langle c, a \cdot D(b)-D(a b)\rangle=-\langle c, D(a) \cdot b\rangle=-\langle b c, D(a)\rangle \\
& =-\langle\Delta(b \otimes c), D(a)\rangle=-\left\langle b \otimes c, \Delta^{*}(D(a))\right\rangle .
\end{aligned}
$$

Hence, $\Delta^{*}(D(a))=\operatorname{ad}_{(-f)}(a)$ and

$$
\begin{aligned}
D(a) & =w^{*}-\lim _{\alpha}\left(\rho_{\alpha}^{*} \circ \Delta^{*}\right)(D(a))=w^{*}-\lim _{\alpha} \rho_{\alpha}^{*}\left(\operatorname{ad}_{-f}(a)\right) \\
& =w^{*}-\lim _{\alpha}\left(\rho_{\alpha}^{*}(f \cdot a-a \cdot f)\right)=w^{*}-\lim _{\alpha}\left(\rho_{\alpha}^{*}(f) \cdot a-a \cdot \rho_{\alpha}^{*}(f)\right) \\
& =w^{*}-\lim _{\alpha} \operatorname{ad}_{\rho_{\alpha}^{*}(-f)}(a) .
\end{aligned}
$$

Therefore, $D$ is $w^{*}$-approximately inner.

Corollary 3.12. Every commutative approximately biprojective Banach algebra is weakly amenable (that is, every derivation $D: \mathcal{A} \rightarrow \mathcal{A}^{*}$ is inner).

Corollary 3.13. Let $\mathcal{A}$ be a boundedly approximately biprojective Banach algebra. Then $\mathcal{A}$ is weakly amenable.

PRoof. If the net $\left(\rho_{\alpha}\right)$ is bounded in the proof of Proposition 3.11, we can find a subnet of $\left(\rho_{\alpha}^{*}(-f)\right)$ which converges to an element $g \in \mathcal{A}^{*}$ in the $w^{*}$-topology, and so $D=\operatorname{ad}_{g}$.

\section{Examples}

In this section, we explore the notion of (bounded) approximate biprojectivity for $\mathrm{C}^{*}$-algebras and Banach algebras related to locally compact groups and inverse semigroups.

By [15, Examples C.1.2(C)], for a measure space $(X, \Sigma, \mu)$, the Banach space $L^{p}(X, \Sigma, \mu)(1 \leq p \leq \infty)$ has the bounded approximation property. So we have the following example.

EXAMPLE 4.1. Let $S$ be an infinite nonempty set and consider $l^{2}(S)$ with the pointwise multiplication. Then by [17, Example], $l^{2}(S)$ is approximately biprojective. We claim that it is not boundedly approximately biprojective. Otherwise, since $l^{2}(S)$ has the bounded approximation property, by [1, Theorem 3.6(A)], it is biflat and thus biprojective since $l^{2}(S)$ is a Hilbert space. This is a contradiction because $l^{2}(S)$ is not biprojective by [17, Example].

ExAmple 4.2. We have the following examples.

(i) Let $G$ be a locally compact group. Then $L^{1}(G)$, the group algebra of $G$, is boundedly approximately biprojective if and only if $G$ is amenable (Johnson's theorem). 
(ii) Let $S$ be an inverse semigroup. Then the semigroup algebra $l^{1}(S)$ is boundedly approximately biprojective if and only if $S$ is uniformly locally finite and every maximal subgroup of $S$ is amenable [14, Theorem 3.7].

Using Proposition 3.4, in the following example, we verify the approximate biprojectivity of Segal algebras on abelian locally compact groups and compact groups.

ExAmple 4.3. We have the following examples.

(i) Every Segal algebra on an abelian locally compact group is approximately biprojective [10, Proposition 4.4]. Note that, for example, if $G$ is an abelian infinite compact group, then the Segal algebra $L^{2}(G)$ is not biprojective [17, Example]. Since $L^{2}(G)$ is a Hilbert space, it is not biflat, and by [1, Theorem 3.6(A)] is not boundedly approximately biprojective.

(ii) Every Segal algebra on a compact group is approximately biprojective [10, Theorem 4.5].

Regarding the approximate biprojectivity of the Fourier algebra on a locally compact group, by Corollary 3.6 we have the following example.

ExAmple 4.4. Let $G$ be a locally compact group and $A(G)$ be its Fourier algebra.

(i) If $A(G)$ has an approximate identity and $G$ contains an open abelian subgroup, then $A(G)$ is approximately biprojective [9, Corollary 3.2].

(ii) If $G$ is discrete, then $A(G)$ is approximately biprojective if and only if $A(G)$ has an approximate identity [9, Corollary 3.2].

It is known that $A(G)$ has a bounded approximate identity if and only if $G$ is amenable (Leptin's theorem, [15, Theorem 7.1.3]). It is also shown in [11, Theorem 2.1] that $A\left(\mathbb{F}_{2}\right)$ has an approximate identity (not bounded), where $\mathbb{F}_{2}$ is the free group in two generators, and so $A\left(\mathbb{F}_{2}\right)$ is approximately biprojective.

Using Corollary 3.7, we have the following example.

EXAMPLE 4.5. Let $G$ be a locally compact group.

(i) If $G$ is discrete, then $l^{1}(G)$ is approximately biprojective if and only if $G$ is amenable [7, Theorem 3.2].

(ii) The measure algebra of $G, M(G)$, is approximately biprojective if and only if $G$ is discrete and amenable; see [3] and [7, Theorem 3.1].

(iii) Let $M(G)^{* *}$, the second dual of $M(G)$ with the first Arens product, be approximately biprojective. Then it is approximately amenable by Corollary 3.7. Since every homomorphic image of an approximately amenable Banach algebra is approximately amenable [7, Proposition 2.2], we see that $l^{1}(G)^{* *}$ must be approximately amenable, and thus $G$ must be finite by [7, Theorem 3.3].

(iv) If $\operatorname{LUC}(G)$ denotes the Banach algebra of left uniformly continuous functions on $G$ and $E$ is a right identity of $L^{1}(G)^{* *}$ with $\|E\|=1$, then by [7, Corollary 3.1], the unital Banach algebra $\operatorname{LUC}(G)^{*} \cong E L^{1}(G)^{* *}$ is approximately biprojective if and only if $G$ is finite. 
Corollary 3.10 gives the following example.

EXAmple 4.6. We have the following examples.

(i) $\mathrm{A} \mathrm{C}^{*}$-algebra is boundedly approximately biprojective if and only if it is nuclear, by [15, Corollary 6.5.12].

(ii) Let $G$ be a locally compact group. Then $A(G)$ is boundedly approximately biprojective if and only if $G$ has an abelian subgroup of finite index [6, Theorem 2.3].

Bounded approximate biprojectivity of the second dual of the group algebra of a locally compact group is characterised by Corollary 3.13.

EXAmple 4.7. Let $G$ be a locally compact group. Then $L^{1}(G)^{* *}$ is boundedly approximately biprojective if and only if $G$ is finite, by [4, Theorem 11.17].

\section{Property $(\mathbb{B})$, nilpotent ideals and approximate biprojectivity}

It is well known that if a Banach space $X$ has the approximation property and $\iota: \mathcal{X} \rightarrow \mathcal{Y}$ is an embedding, then for each Banach space $\mathcal{Z}$ the mapping $\iota \otimes I_{\mathcal{Z}}$ : $\mathcal{X} \hat{\otimes} \mathcal{Z} \rightarrow \mathcal{Y} \hat{\otimes} \mathcal{Z}$ is injective.

Proposition 5.1. Let $\mathcal{X}$ be a bounded approximately complemented Banach subspace of a Banach space $\mathcal{Y}$, and let $\iota: \mathcal{X} \rightarrow \mathcal{Y}$ be the inclusion map. For a Banach space $\mathcal{Z}$, if $\left(q_{n}\right) \subseteq \mathcal{X} \hat{\otimes} \mathcal{Z}$ is a sequence such that $\left(\iota \otimes I_{\mathcal{Z}}\right)\left(q_{n}\right) \rightarrow 0$, then $q_{n} \rightarrow 0$.

Proof. Let $q_{n}=\sum_{m=1}^{\infty} x_{m}^{n} \otimes z_{m}^{n}$. We can assume that for each $n \in \mathbb{N}, x_{m}^{n} \rightarrow 0$ and $\sum_{m=1}^{\infty}\left\|z_{m}^{n}\right\|_{\mathcal{Z}}<1$. Take $\epsilon>0$. Since for each $n$ the set $\left\{x_{m}^{n}\right\} \cup\{0\}$ is compact, there exists a bounded sequence $S_{n}: \mathcal{Y} \rightarrow \mathcal{X}$ such that $\left\|S_{n}\left(x_{m}^{n}\right)-x_{m}^{n}\right\|_{y}<\epsilon$ for all $m$. Then

$$
\begin{aligned}
\left\|q_{n}\right\|_{\mathcal{X} \hat{\otimes} \mathcal{Z}} & \leq\left\|\sum_{m=1}^{\infty} x_{m}^{n} \otimes z_{m}^{n}\right\|_{\mathcal{X} \hat{\mathcal{Z}}} \\
& \leq\left\|\sum_{m=1}^{\infty} S_{n}\left(x_{m}^{n}\right) \otimes z_{m}^{n}\right\|_{\mathcal{X} \hat{\otimes} \mathcal{Z}}+\epsilon \\
& \leq\left\|\left(S_{n} \otimes I_{\mathcal{Z}}\right)\left(\sum_{m=1}^{\infty} x_{m}^{n} \otimes z_{m}^{n}\right)\right\|_{\mathcal{X} \hat{\otimes} \mathcal{Z}}+\epsilon \\
& \leq\left\|S_{n}\right\|\left\|\left(\iota \otimes I_{\mathcal{Z}}\right)\left(\sum_{m=1}^{\infty} x_{m}^{n} \otimes z_{m}^{n}\right)\right\|_{\mathcal{Y} \hat{\otimes} \mathcal{Z}}+\epsilon .
\end{aligned}
$$

It follows that $q_{n} \rightarrow 0$.

Motivated by Proposition 5.1 and the discussed fact before it, we give the following definition, which will help us to get the main results of this paper.

Definition 5.2. Let $\mathcal{I}$ be a closed ideal in a Banach algebra $\mathcal{A}$, and let $\iota: \mathcal{I} \rightarrow \mathcal{A}$ be the inclusion map. We say that $\mathcal{I}$ has the property $(\mathbb{B})$ if the following assertion is true. 
(B) If $\left(q_{n}\right) \subseteq \mathcal{I} \hat{\otimes} \mathcal{A} / \mathcal{J}$ is a sequence such that $\left(\iota \otimes I_{\mathcal{H} / \mathcal{J}}\right)\left(q_{n}\right) \rightarrow 0$ for some closed ideal $\mathcal{J}$ of $\mathcal{A}$ with $\mathcal{J} \subseteq \mathcal{I}$ and $\mathcal{J}^{2}=0$, then $q_{n} \rightarrow 0$.

We remark that there are many Banach algebras having ideals satisfying property $(\mathbb{B})$. For example, in semi-prime Banach algebras (that is, having no nonzero closed ideal $\mathcal{I}$ with $\mathcal{I}^{2}=0$ ) every closed ideal has the property $(\mathbb{B})$. Also, every boundedly approximately complemented closed ideal in a Banach algebra has the property $(\mathbb{B})$ (Proposition 5.1). Therefore, every complemented closed ideal, in particular every finite-dimensional and every finite-codimensional ideal, has the property $(\mathbb{B})$.

The following lemma and theorem are parallel to Lemma 2 and the theorem of [17], respectively. In the proofs we use similar techniques.

Lemma 5.3. Suppose that $\mathcal{A}$ is an approximately biprojective Banach algebra, $\mathcal{J}$ and $\mathcal{N}$ are closed ideals in $\mathcal{A}$ such that $\mathcal{J} \subseteq \mathcal{N}$ and $\mathcal{N} \mathcal{J}=\{0\}(\mathcal{J N}=\{0\})$. Suppose also that $\mathcal{N}$ has the property $(\mathbb{B})$, and $\mathcal{A}$ has a left (right) approximate identity for $\mathcal{J}$, or $\mathcal{A}$ has an element which is not a left (right) zero divisor. Then $\mathcal{J} \mathcal{A}=\{0\}(\mathcal{A} \mathcal{J}=\{0\})$.

Proof. We show that $\mathcal{J} \mathcal{A}=\{0\}$, the other case is similar. Let $\left(\rho_{\alpha}\right)_{\alpha \in \Gamma} \subseteq \mathcal{B}(\mathcal{A}, \mathcal{A} \hat{\otimes} \mathcal{A})$ be a net satisfying Definition 3.1. Let $\iota: \mathcal{N} \rightarrow \mathcal{A}$ and $q: \mathcal{A} \rightarrow \mathcal{A} / \mathcal{J}$ be the inclusion and quotient maps, respectively. Since $\mathcal{N} \mathcal{J}=0$, the operator $p: \mathcal{N} \hat{\otimes} \mathcal{A} / \mathcal{J} \rightarrow \mathcal{N}$ determined by $p(b \otimes(c+\mathcal{J}))=b c$ is well defined.

Suppose in contrast that $\mathcal{J} \mathcal{A} \neq\{0\}$. Using the ideal $\mathcal{J}$ (note that $\mathcal{J}^{2}=\{0\}$ ), we show that $\mathcal{N}$ does not have the property $(\mathbb{B})$. Since $\mathcal{J} \mathcal{A} \neq\{0\}$, there exist $a \in \mathcal{A}$ and $j \in \mathcal{J}$ with $j a \neq 0$. We can find $u \in \mathcal{A}$ such that $u j a \neq 0$, by our assumption. Let $\mathcal{L}_{b}$ and $\mathcal{R}_{b}: \mathcal{A} \rightarrow \mathcal{N}$ be the left and right multiplication maps by $b \in \mathcal{N}$, respectively. For each $\alpha$ set $v_{\alpha}=\left(\mathcal{L}_{(u j)} \otimes q\right) \circ \rho_{\alpha}(a)$ which belongs to $\mathcal{N} \hat{\otimes} \mathcal{A} / \mathcal{J}$. Since $q \circ \iota \circ \mathcal{R}_{(j a)}=0$, by condition (iii) of Definition 3.1,

$$
\begin{aligned}
\lim _{\alpha}\left(I_{\mathcal{A}} \otimes q\right)\left(\rho_{\alpha}(u j a)\right) & =\lim _{\alpha}\left(I_{\mathcal{A}} \otimes q\right)\left(\rho_{\alpha}(u) j a\right) \\
& =\lim _{\alpha}\left(I_{\mathcal{A}} \otimes q\right) \circ\left(I_{\mathcal{A}} \otimes \iota\right) \circ\left(I_{\mathcal{A}} \otimes \mathcal{R}_{(j a)}\right) \circ \rho_{\alpha}(u) \\
& =\lim _{\alpha}\left(I_{\mathcal{A}} \otimes\left(q \circ \iota \circ \mathcal{R}_{(j a)}\right) \circ \rho_{\alpha}(u)\right. \\
& =0
\end{aligned}
$$

and, thus, by condition (ii) of Definition 3.1,

$$
\begin{aligned}
\lim _{\alpha}\left(\iota \otimes I_{\mathcal{H} / \mathcal{J}}\right)\left(v_{\alpha}\right) & =\lim _{\alpha}\left(\iota \otimes I_{\mathcal{A} / \mathcal{J}}\right) \circ\left(I_{\mathcal{N}} \otimes q\right) \circ\left(\mathcal{L}_{(u j)} \otimes I_{\mathcal{H}}\right) \circ \rho_{\alpha}(a) \\
& =\lim _{\alpha}\left(I_{\mathcal{A}} \otimes q\right) \circ\left(\iota \otimes I_{\mathcal{A}}\right) \circ\left(\mathcal{L}_{(u j)} \otimes I_{\mathcal{A}}\right) \circ \rho_{\alpha}(a) \\
& =\lim _{\alpha}\left(I_{\mathcal{A}} \otimes q\right)\left(u j \rho_{\alpha}(a)\right) \\
& =\lim _{\alpha}\left(I_{\mathcal{A}} \otimes q\right)\left(\rho_{\alpha}(u j a)\right) \\
& =0 .
\end{aligned}
$$


This implies that

$$
\forall n \in \mathbb{N} \exists \alpha_{n} \in \Gamma \text { such that } \forall \alpha \in \Gamma \text { if } \alpha \geq \alpha_{n} \text {, then }\left\|\left(\iota \otimes I_{\mathcal{H} / \mathcal{J}}\right)\left(v_{\alpha}\right)\right\|<\frac{1}{n} .
$$

Now

$$
\lim _{\alpha} p\left(v_{\alpha}\right)=\lim _{\alpha}\left(\mathcal{L}_{(u j)} \circ \Delta \circ \rho_{\alpha}\right)(a)=\lim _{\alpha}\left(u j \Delta \circ \rho_{\alpha}(a)\right)=u j a \neq 0,
$$

and so $\lim _{\alpha} v_{\alpha} \neq 0$. Therefore, there exists $\epsilon>0$ such that for each $\alpha \in \Gamma$ there is a $\beta=\beta(\alpha)$ with $\beta \geq \alpha$ and $\left\|p\left(v_{\beta}\right)\right\| \geq \epsilon$. Thus, for each $n \in \mathbb{N}$, there is a $\beta_{n} \geq \alpha_{n}$ such that $\left\|p\left(v_{\beta_{n}}\right)\right\| \geq \epsilon$, which shows that $\left(p\left(v_{\beta_{n}}\right)\right)$ and hence $\left(v_{\beta_{n}}\right)$ does not converge to 0 . But, by $(5.1),\left\|\left(\iota \otimes I_{\mathcal{A} / \mathcal{J}}\right)\left(v_{\beta_{n}}\right)\right\|<1 / n$, and thus $\left(\iota \otimes I_{\mathcal{H} / \mathcal{J}}\right)\left(v_{\beta_{n}}\right) \rightarrow 0$. This shows that $\mathcal{N}$ does not have the property $(\mathbb{B})$, a contradiction.

Theorem 5.4. Suppose that $\mathcal{A}$ is an approximately biprojective Banach algebra. If $\mathcal{A}$ has both left and right approximate identities, then $\mathcal{A}$ cannot have a nonzero nilpotent ideal with the property $(\mathbb{B})$.

Proof. Let $\mathcal{N}$ be a nonzero nilpotent ideal in $\mathcal{A}$ with $\operatorname{Nil}(\mathcal{N})=n+1$ and with the property $(\mathbb{B})$. Let $\mathcal{J} \subseteq \mathcal{N}$ be the closed linear span of $\mathcal{N}^{n}$ which is a nonzero closed ideal in $\mathcal{A}$ and $\mathcal{N} \mathcal{J}=\{0\}$. Since $\mathcal{A}$ has a left approximate identity, by Lemma 5.3, $\mathcal{J} \mathcal{A}=\{0\}$ and since $\mathcal{A}$ has a right approximate identity, we obtain $\mathcal{J}=\overline{\mathcal{J} \mathscr{A}}=\{0\}$ which is a contradiction.

Corollary 5.5. Let $\mathcal{A}$ be an approximately biprojective Banach algebra with both left and right approximate identities. Then $\mathcal{A}$ cannot have a nonzero nilpotent ideal which is boundedly approximately complemented. In particular, $\mathcal{A}$ has no finite-dimensional nilpotent ideal, and $s o \operatorname{rad}(\mathcal{A})$ cannot be finite-dimensional.

Since every pseudo-amenable Banach algebra has an approximate identity and also every approximately amenable Banach algebra has both left and right approximate identities [7, Lemma 2.2], we obtain the following corollary.

Corollary 5.6. Let $\mathcal{A}$ be an approximately amenable or a pseudo-amenable Banach algebra. Then by Proposition 3.4 and Corollary 5.5:

(i) $\mathcal{A}$ cannot have a nonzero nilpotent ideal with the property $(\mathbb{B})$;

(ii) nilpotent ideals cannot be boundedly approximately complemented in $\mathcal{A}$;

(iii) $\mathcal{A}$ has no finite-dimensional nilpotent ideal;

(iv) $\operatorname{rad}(\mathcal{A})$ cannot be finite-dimensional.

The next theorem is another version of Proposition 1.1 and the proof is similar.

Theorem 5.7. Let $\mathcal{A}$ be an approximately biprojective Banach algebra and $\mathcal{N}$ be a nonzero nilpotent ideal in $\mathcal{A}$. Suppose that there exists an element $u \in \mathcal{A}$ such that for some $0 \neq b \in \mathcal{N}, b u=u b \neq 0$. Then $\mathcal{N}$ does not have the property $(\mathbb{B})$.

Proof. Let $\mathcal{J}$ be the closed linear span of $\mathcal{N}^{n}$, where $n=\operatorname{Nil}(\mathcal{N})-1$. Let $\left(\rho_{\alpha}\right)_{\alpha \in \Gamma} \subseteq$ $\mathcal{B}(\mathcal{A}, \mathcal{A} \hat{\otimes} \mathcal{A})$ be a net satisfying Definition 3.1. Let $\iota: \mathcal{N} \rightarrow \mathcal{A}$ and $q: \mathcal{A} \rightarrow \mathcal{A} / \mathcal{J}$ be 
the inclusion and quotient maps, respectively. Let also $\Delta_{\mathcal{N}}: \mathcal{N} \hat{\otimes} \mathcal{A} \rightarrow \mathcal{N}$ be the map determined by $\Delta_{\mathcal{N}}(c \otimes a)=c a$ for all $c \in \mathcal{N}$ and $a \in \mathcal{A}$. Note that $\iota \circ \Delta_{\mathcal{N}}=\Delta \circ\left(\iota \otimes I_{\mathcal{A}}\right)$. Clearly $\mathcal{N} \mathcal{J}=0$, and thus $\Delta_{\mathcal{N}}$ factors through $\mathcal{N} \hat{\otimes} \mathcal{A} / \mathcal{J}$ as $\Delta_{\mathcal{N}}=p \circ\left(I_{\mathcal{N}} \otimes q\right)$, where $p: \mathcal{N} \hat{\otimes} \mathcal{A} / \mathcal{J} \rightarrow \mathcal{N}$ is the map determined by $p(c \otimes(a+\mathcal{J}))=c a$.

Now for $c \in \mathcal{N}$, let $\mathcal{L}_{c}$ and $\mathcal{R}_{c}: \mathcal{A} \rightarrow \mathcal{N}$ be the left and right multiplication maps by $c$, respectively. Then $\Delta_{\mathcal{N}} \circ\left(\mathcal{L}_{c} \otimes I_{\mathcal{A}}\right)=\mathcal{L}_{c} \circ \Delta$. Take $0 \neq b \in \mathcal{N}$ and $u \in \mathcal{A}$ such that $b u=u b \neq 0$, and set $v_{\alpha}=\left(\left(I_{\mathcal{N}} \otimes q\right) \circ\left(\mathcal{L}_{b} \otimes I_{\mathcal{A}}\right) \circ \rho_{\alpha}\right)(u)$ which belongs to $\mathcal{N} \hat{\otimes} \mathcal{A} / \mathcal{J}$. Then

$$
p\left(v_{\alpha}\right)=\left(\Delta_{\mathcal{N}} \circ\left(\mathcal{L}_{b} \otimes I_{\mathcal{A}}\right) \circ \rho_{\alpha}\right)(u)=\left(\mathcal{L}_{b} \circ \Delta \circ \rho_{\alpha}\right)(u)=b\left(\Delta \circ \rho_{\alpha}\right)(u),
$$

and so $\lim _{\alpha} p\left(v_{\alpha}\right)=\lim _{\alpha} b\left(\Delta \circ \rho_{\alpha}\right)(u)=b u$. Since $u$ commutes with $b$ and $\left(\rho_{\alpha}\right)$ satisfies conditions (ii) and (iii) of Definition 3.1, we have $\lim _{\alpha}\left(b \cdot \rho_{\alpha}(u)-\rho_{\alpha}(u) \cdot b\right)=0$ and thus

$$
\begin{aligned}
& \lim _{\alpha}\left(\left(\left(I_{\mathcal{A}} \otimes q\right) \circ\left(\iota \otimes I_{\mathcal{A}}\right) \circ\left(\mathcal{L}_{b} \otimes I_{\mathcal{A}}\right) \circ \rho_{\alpha}\right)(u)\right. \\
& \left.\quad-\left(\left(I_{\mathcal{A}} \otimes q\right) \circ\left(I_{\mathcal{A}} \otimes \iota\right) \circ\left(I_{\mathcal{A}} \otimes \mathcal{R}_{b}\right) \circ \rho_{\alpha}\right)(u)\right)=0 .
\end{aligned}
$$

Since $q \circ \iota \circ \mathcal{R}_{b}=0$, for each $\alpha \in \Gamma$,

$$
\left(I_{\mathcal{A}} \otimes q\right) \circ\left(I_{\mathcal{A}} \otimes \iota\right) \circ\left(I_{\mathcal{A}} \otimes \mathcal{R}_{b}\right) \circ \rho_{\alpha}(u)=\left(I_{\mathcal{A}} \otimes\left(q \circ \iota \circ \mathcal{R}_{b}\right)\right) \circ \rho_{\alpha}(u)=0,
$$

whence

$$
\lim _{\alpha}\left(\iota \otimes I_{\mathcal{A} / \mathcal{J}}\right)\left(v_{\alpha}\right)=\lim _{\alpha}\left[\left(I_{\mathcal{A}} \otimes q\right) \circ\left(\iota \otimes I_{\mathcal{A}}\right) \circ\left(\mathcal{L}_{b} \otimes I_{\mathcal{A}}\right) \circ \rho_{\alpha}\right](u)=0 .
$$

Now as in the proof of Lemma 5.3 we see that $\mathcal{N}$ does not have the property $(\mathbb{B})$.

Corollary 5.8. In the setting of this theorem, $\mathcal{N}$ cannot be boundedly approximately complemented in $\mathcal{A}$.

Remark 5.9. Note that there are many Banach algebras that have such $u$ as in Theorem 5.7. For example, if $\mathcal{A}$ has a unit or has a central approximate identity or has a central element that is not a zero divisor, then we have such an element.

Theorem 5.10. Let $\mathcal{A}$ be an approximately biprojective Banach algebra and $\mathcal{N}$ be a nonzero nilpotent ideal in $\mathcal{A}$ with $\operatorname{Nil}(\mathcal{N})>2$. Then $\mathcal{N}$ does not have the property $(\mathbb{B})$, and so cannot be boundedly approximately complemented in $\mathcal{A}$.

Proof. Let $a, b \in \mathcal{N}$ be such that $b a \neq 0$. Using the notation in the proof of Theorem 5.7, change the role of $u$ by $a$, we have $v_{\alpha}=\left(\left(I_{\mathcal{N}} \otimes q\right) \circ\left(\mathcal{L}_{b} \otimes I_{\mathcal{A}}\right) \circ \rho_{\alpha}\right)(a)$ which belongs to $\mathcal{N} \hat{\otimes} \mathcal{A} / \mathcal{J}$ and $p\left(v_{\alpha}\right)=b\left(\Delta \circ \rho_{\alpha}\right)(a)$. So $\lim _{\alpha} p\left(v_{\alpha}\right)=b a \neq 0$. Since $\left(\rho_{\alpha}\right)$ satisfies conditions (ii) and (iii) of Definition 3.1, we have $\lim _{\alpha}\left(b \cdot \rho_{\alpha}(a)-\right.$ $\left.\rho_{\alpha}(b) \cdot a\right)=0$, and so

$$
\begin{aligned}
& \lim _{\alpha}\left(\left(\left(I_{\mathcal{A}} \otimes q\right) \circ\left(\iota \otimes I_{\mathcal{A}}\right) \circ\left(\mathcal{L}_{b} \otimes I_{\mathcal{A}}\right) \circ \rho_{\alpha}\right)(a)\right. \\
& \left.\quad-\left(\left(I_{\mathcal{A}} \otimes q\right) \circ\left(I_{\mathcal{A}} \otimes \iota\right) \circ\left(I_{\mathcal{A}} \otimes \mathcal{R}_{a}\right) \circ \rho_{\alpha}\right)(b)\right)=0 .
\end{aligned}
$$


Since $q \circ \iota \circ \mathcal{R}_{a}=0$, for each $\alpha \in \Gamma$,

$$
\left(I_{\mathcal{A}} \otimes q\right) \circ\left(I_{\mathcal{A}} \otimes \iota\right) \circ\left(I_{\mathcal{A}} \otimes \mathcal{R}_{a}\right) \circ \rho_{\alpha}(b)=\left(I_{\mathcal{A}} \otimes\left(q \circ \iota \circ \mathcal{R}_{a}\right)\right) \circ \rho_{\alpha}(b)=0,
$$

and so

$$
\lim _{\alpha}\left(\iota \otimes I_{\mathcal{A} / \mathcal{J}}\right)\left(v_{\alpha}\right)=\lim _{\alpha}\left(\left(I_{\mathcal{A}} \otimes q\right) \circ\left(\iota \otimes I_{\mathcal{A}}\right) \circ\left(\mathcal{L}_{b} \otimes I_{\mathcal{A}}\right) \circ \rho_{\alpha}\right)(a)=0
$$

Hence, as in the proof of Lemma 5.3, $\mathcal{N}$ does not have the property $(\mathbb{B})$ and, thus, by Proposition 5.1, $\mathcal{N}$ cannot be boundedly approximately complemented in $\mathcal{A}$.

The following corollary is a direct consequence of Theorems 3.3 and 5.10.

Corollary 5.11. Let $\mathcal{A}$ be a biflat Banach algebra and $\mathcal{N}$ be a nonzero nilpotent ideal in $\mathcal{A}$ with $\operatorname{Nil}(\mathcal{N})>2$. Then $\mathcal{N}$ cannot be boundedly approximately complemented in $\mathcal{A}$.

\section{References}

[1] O. Yu. Aristov, 'On approximation of flat Banach modules by free modules', Sbornik. Math. 196(11) (2005), 1553-1583.

[2] P. C. Curtis Jr and R. J. Loy, 'The structure of amenable Banach algebras', J. Lond. Math. Soc. 40(2) (1989), 89-104.

[3] H. G. Dales, F. Ghahramani and A. Yu. Helemskii, 'The amenability of measure algebras', J. Lond. Math. Soc. 66(1) (2002), 213-226.

[4] H. G. Dales, A. T. Lau and D. Strauss, 'Banach algebras on semigroups and on their compactifications', Mem. Amer. Math. Soc. 205 (2010), 1-165.

[5] H. G. Dales, R. J. Loy and Y. Zhang, 'Approximate amenability for Banach sequence algebras', Studia Math. 177 (2006), 81-96.

[6] B. E. Forrest and V. Runde, 'Amenability and weak amenability of the Fourier algebra', Math. Z. 250(4) (2005), 731-744.

[7] F. Ghahramani and R. J. Loy, 'Generalized notions of amenability', J. Funct. Anal. 208 (2004), 229-260.

[8] F. Ghahramani, R. J. Loy and Y. Zhang, 'Generalized notions of amenability, II', J. Funct. Anal. 254 (2008), 1776-1810.

[9] F. Ghahramani and R. Stokke, 'Approximate and pseudo-amenability of the Fourier algebra', Indiana Univ. Math. J. 56 (2007), 909-930.

[10] F. Ghahramani and Y. Zhang, 'Pseudo-amenable and pseudo-contractible Banach algebras', Math. Proc. Cambridge Philos. Soc. 142 (2007), 111-123.

[11] U. Haagerup, 'An example of a nonnuclear $\mathrm{C}^{*}$-algebra, which has the metric approximation property', Invent. Math. 50 (1978/79), 279-293.

[12] A. Ya. Helemskii, The Homology of Banach and Topological Algebras (Kluwer, Dordrecht, 1989).

[13] R. J. Loy and G. A. Willis, 'The approximation property and nilpotent ideals in amenable Banach algebras', Bull. Aust. Math. Soc. 49 (1994), 341-346.

[14] P. Ramsden, 'Biflatness of semigroup algebras', Semigroup Forum 79 (2009), 515-530.

[15] V. Runde, Lectures on Amenability, Lecture Notes in Mathematics, 1774 (Springer, Berlin, 2002).

[16] E. Semei, N. Spronk and R. Stokke, 'Biflatness and Pseudo-Amenability of Segal algebras', Canad. J. Math. 62(4) (2010), 845-869. 
[17] Y. Zhang, 'Nilpotent ideals in a class of Banach algebras', Proc. Amer. Math. Soc. 127(11) (1999), 3237-3242.

[18] Y. Zhang, 'Approximate complementation and its application in studying ideals of Banach algebras', Math. Scand. 92 (2003), 301-308.

HASAN POURMAHMOOD-AGHABABA, Department of Mathematics, University of Tabriz, Tabriz, Iran

and

School of Mathematics, Institute for Research in Fundamental Sciences (IPM), PO Box 19395-5746, Tehran, Iran

e-mail: h_p_aghababa@tabrizu.ac.ir, h_pourmahmood@yahoo.com 